\title{
Identification of the two KIT isoforms and their expression status in canine hemangiosarcomas
}

\author{
Yi-Chen Chen ${ }^{1,2}$, Jiunn-Wang Liao ${ }^{2}$, Wei-Li Hsu ${ }^{3^{*}}$ and Shih-Chieh Chang ${ }^{1,4^{*}}$
}

\begin{abstract}
Background: KIT is a tyrosine kinase growth factor receptor. High expression of KIT has been found in several tumors including canine hemangiosarcoma (HSA). This study investigated the correlation of KIT expression and c-kit sequence mutations in canine HSAs and benign hemangiomas (HAs).

Results: Immunohistochemistry (IHC) staining confirmed KIT expression in $94.4 \%(34 / 36)$ of HSAs that was significantly higher than $0 \%$ in HAs (0/16). Sequencing the entire c-kit coding region of HSAs and normal canine cerebellums (NCCs) revealed GNSK-deletion in exon 9. As for exon 9 genotyping by TA-cloning strategy, GNSKdeletion c-kit accounted for $48.6 \%$ (68/140) colonies amplified from12 KIT-positive HSAs, a significantly higher frequency than $14.1 \%(9 / 64)$ of colonies amplified from six NCCs.

Conclusions: Due to the distinct expression pattern revealed by IHC, KIT might be used to distinguish benign or malignant vascular endothelial tumors. Moreover, the high incidence of GNSK-deletion c-kit in canine HSAs implicates KIT isoforms as possibly participating in the tumorigenesis of canine HSAs.
\end{abstract}

Keywords: Canine, Hemangiosarcoma, c-kit, GNSK-deletion, Isoform, KIT

\section{Background}

Hemangiosarcomas (HSAs) are highly malignant tumors of vascular endothelial origin, which occur more frequently in dogs than other domestic species and are characterized by a high fatality rate $[1,2]$. Surgical excision remains the primary treatment for most dogs with HSAs [3]. However, owing to a poor outcome by surgery alone, adjuvant chemotherapy is suggested for canine HSAs [4].

KIT protein, encoded by proto-oncogene $c$-kit, is a tyrosine kinase growth factor receptor for stem cell factor (SCF). Generally, KIT is activated by autophosphorylation upon the binding of its ligand SCF [5]; however it is constitutively expressed in a number of cells including mast cells, and hematopoietic stem cells

\footnotetext{
* Correspondence: whsu@dragon.nchu.edu.tw; scchang@dragon.nchu.edu.tw ${ }^{3}$ Graduate Institute of Microbiology and Public Health, College of Veterinary Medicine, National Chung Hsing University, 250 Kuo-Kuang Road, Taichung 40227, Taiwan

'Department of Veterinary Medicine, College of Veterinary Medicine, National Chung Hsing University, 250 Kuo-Kuang Road, Taichung 40227, Taiwan Full list of author information is available at the end of the article
}

[6]. In humans, KIT is often expressed in angiosarcomas, but it is not detected in most benign vascular tumors, and KIT positivity is more likely related to an immature phenotype [7]. As with human tissues, KIT is expressed in a large proportion of canine HSAs [8]. It has been demonstrated that activating mutations of $c$-kit typically confer constitutional KIT phosphorylation leading to downstream activation independent of ligand binding [9]. Among the 21 exons, the common activating mutations are located in exons 11 and 17, coding for juxtamembrane domain and tyrosine kinase domain of KIT, respectively [10-12]. Gain-of-function mutations have been proposed to contribute to neoplastic growth of several tumor mast cell lines [13]. Sequence analysis of canine mast cell tumors (MCTs) identified several mutations in exons 8, 9, 11, and 17 of $c$-kit RNA [14]. In contrast to MCTs, no mutation was found in these two exons (11 and 17) of c-kit gene in KIT-positive neoplasms of vascular cell origin [7].

Imatinib (Gleevec; Novartis), the tyrosine kinase inhibitor that targets activating mutations of $c$-kit, has been used in patients with gastrointestinal stromal 
tumors (GIST) harboring a c-kit exon 11 mutation. Decreases in tumor mass and increases of survival time have been reported $[15,16]$.

At present, $c$-kit gene polymorphism was detected in various canine tumors [17], but information of mutations or the impact of the $c$-kit gene in canine HSAs was limited. Since $c$-kit gene mutations could influence the expression level of KIT, and also the sensitivity of kinase inhibitor (such as imatinib mesylate) treatment [18], a systemic investigation of the mutation status of $c$-kit would provide prognostic information for tumor pathogenesis and also for the clinical response of patients under imatinib therapy [19]. Hence, this study aimed to evaluate the contribution of KIT expression and also genetic variations in the malignancy of canine HSAs.

\section{Methods}

\section{Samples}

Dogs with cutaneous hemangiomas (HAs) or splenic/cutaneous HSAs presented to the Veterinary Medical Teaching Hospital from 2005 to 2012 were enrolled. Medical records included breed, age, sex, number of tumors, tumor size, anatomical location, clinical history, physical examination, complete blood count, serum biochemical profile, thoracic and abdominal radiographs, abdominal ultrasonography, fine-needle cytological examination and needle core biopsy or surgical excision for histological examination. All HSAs were staged according to the World Health Organization staging system for canine splenic HSAs [9] and cutaneous HSAs [20]. The protocol of study was approved by the Institutional Animal Care and Use Committee of National Chung Hsing University [IACUC Number: 102-70].

In total, 52 specimens ( 16 HAs by surgical excision, 24 HSAs by surgical excision and 12 cutaneous HSAs by needle core biopsy) were included in this study. For histologic examination, specimens were fixed in neutralbuffered $10 \%$ formalin overnight, and then processed routinely. Sections were stained with haematoxylin and eosin. Histologic grading of HSAs was assigned by cumulative scores of differentiation, mitotic rate (the number of mitotic figures per 10 high magnification fields) and percentage of necrosis [21].

\section{Immunohistochemistry (IHC) analysis}

Serial sections of formalin-fixed and wax-embedded tissues were deparaffinized and rehydrated. Table 1 lists primary and secondary antibodies and antigen retrieval procedures used in immunohistochemical (IHC) analysis. Antigen retrieval was modified from a previous study [22]. Slides in buffer solution were cooled at room temperature for $20 \mathrm{~min}$, and incubated with peroxidaseblocking reagent (S200389, Dako) for $30 \mathrm{~min}$, and then treated with primary antibodies. In each interval of the following procedures, sections were rinsed with a mixture of Tris-Buffered Saline and Tween-20. Slides were then reacted with secondary antibody followed by incubation of DAB and chromogen (dilution $1 \mu \mathrm{L}$ in $100 \mu \mathrm{L}$ ) from a commercial ChemMate EnVision detection kit (K5007, Dako). Finally, sections were counterstained with Mayer's hematoxylin for 2 min then rinsed with DDW, and incubated with $37 \mathrm{mM}$ ammonia water for $5 \mathrm{~s}$ and rinsed with DDW.

For immunolabelingvon Willebrand factor (vWF), a canine subcutaneous granuloma acted as a positive control, and normal vessels in tissues surrounding the tumor served as an internal positive control [8]. For immunolabeling vimentin or KIT, normal adipose tissues [23] or a normal canine cerebellum (NCC) [24] was used as a positive control respectively. Replacement of primary antibody with antibody dilution buffer served as a negative control.

To score expression, this study followed an immunoreactive score (IRS) system from a previous study [25] in which IRS = SI (staining intensity) x PP (percentage of positive cells). SI was assigned as 0 , negative; 1 , weak; 2 , moderate; and 3, strong. PP was defined as $1,<10 \%$ positive cells; $2,10-50 \%$ positive cells; and $3,>50 \%$ positive cells. Ten high magnification fields were randomly chosen for IRS evaluation. The expression level was classified as 'negative,' 'weak', or 'strong', corresponding to IRS values of

Table 1 Primary and secondary antibodies and antigen retrieval procedures used in IHC analysis of this study

\begin{tabular}{|c|c|c|c|c|c|c|c|}
\hline \multirow[b]{2}{*}{ Antibody } & \multirow[b]{2}{*}{$\begin{array}{l}\mathrm{pAb} / \mathrm{mAb} \\
\text { (clone) }\end{array}$} & \multirow[b]{2}{*}{ Host } & \multirow[b]{2}{*}{ Source } & \multirow[b]{2}{*}{$\begin{array}{l}\text { Antigen retrieval (buffer, } \\
\text { microwave, interval) }\end{array}$} & \multicolumn{3}{|c|}{ Time for incubation } \\
\hline & & & & & Primary Ab & Secondary Ab & $\mathrm{DAB}+$ Chromagen \\
\hline von Willebrand factor & $\mathrm{pAb}$ & Rabbit anti human & Dako & $\begin{array}{l}\text { Citrate buffer (0.01 M pH 6.0), } \\
700 \mathrm{~W}-10 \text { min and 300W-10 min }\end{array}$ & $1: 200,30 \mathrm{~min}$ & $10 \mathrm{~min}$ & $1 \mathrm{~min}$ \\
\hline vimentin & mAb (V9) & Mouse anti human & Dako & $\begin{array}{l}\text { TE buffer (pH 9.0), } 700 \mathrm{~W}-10 \\
\text { min } \\
\text { and } 400 \mathrm{~W}-10 \mathrm{~min}\end{array}$ & $1: 150,1 \mathrm{~h}$ & $10 \mathrm{~min}$ & $10 \mathrm{~min}$ \\
\hline KIT & $\mathrm{pAb}$ & Rabbit anti human & Dako & $\begin{array}{l}\text { TE buffer (pH 9.0),700 W-10 min } \\
\text { and 400W-10 min }\end{array}$ & 1:400, $30 \mathrm{~min}$ & $10 \mathrm{~min}$ & $2 \min$ \\
\hline
\end{tabular}

pAb polyclonal antibody, mAb monoclonal antibody, TE buffer Tris-EDTA buffer solution 
$0-1,2-4$ and 6-9, respectively. For KIT expression, three patterns were determined as pattern I, a membrane associated pattern with little to no cytoplasmic staining; pattern II, a focal (paranuclear or Golgi-like) cytoplasmic pattern with only occasional minor membrane staining, and pattern III, a diffuse cytoplasmic pattern [26].

\section{Identification of $c$-kit coding sequences}

Total RNA extracted with Trizol (Invitrogen) from 12HSAs with KIT-overexpression and from a NCC for amplifying wild-type open reading frame of $c$-kit gene were reverse transcribed into cDNA. A solution of $2 \mu \mathrm{g}$ RNA, $2 \mu \mathrm{L}$ of $5 \mu \mathrm{M}$ random primers, $4 \mu \mathrm{L}$ of $2.5 \mathrm{mM}$ $\mathrm{dNTP}$ and $7 \mu \mathrm{L}$ DDW were incubated at $65{ }^{\circ} \mathrm{C}$ for $5 \mathrm{~min}$, then chilled on ice for $5 \mathrm{~min}$. The mixture of reverse transcriptase solution, which included $1 \mu \mathrm{L}$ of $0.1 \mathrm{M}$ dithiothreitol, $1 \mu \mathrm{L}$ reverse transcriptase (Superscript III, Invitrogen), $4 \mu \mathrm{L}$ of $5 \times$ First Strand Buffer (Invitrogen) and $1 \mu \mathrm{L}$ RNase inhibitor (Promega), was added and heated at $50{ }^{\circ} \mathrm{C}$ for $60 \mathrm{~min}$, then inactivated at $70{ }^{\circ} \mathrm{C}$ for $15 \mathrm{~min}$ and chilled on ice.

Sequencing of the entire $c$-kit coding region was determined by PCR using several sets of primers followed by automated sequencing. Primers were designed according to a previously published canine $c$-kit $\mathrm{cDNA}$ sequence [27]. Sequences, as well as locations of primers, are listed in Table 2.

In general, PCR solution included $2.5 \mu \mathrm{L}$ of $10 \times$ Taq buffer, $2.5 \mu \mathrm{L}$ of $2.5 \mathrm{mM}$ dNTP, $1 \mu \mathrm{L}$ of $5 \mathrm{mM}$ of both sense and anti-sense primer, 0.5 U DreamTaq DNA polymerase (EP0702, Fermentas), $2 \mu \mathrm{l}$ of RT reaction mixture and $15.5 \mu \mathrm{L}$ DDW. PCRwas performed by carrying outinitial denaturation for $5 \mathrm{~min}$ at $95{ }^{\circ} \mathrm{C}$, followed by 40 thermocycles: $1 \mathrm{~min}$ at $95^{\circ} \mathrm{C}$, annealing for $45 \mathrm{~s}$ at 52 to $62{ }^{\circ} \mathrm{C}$ (Table 2) and then $1 \mathrm{~min}$ at $72{ }^{\circ} \mathrm{C}$, and final polymerization for $7 \mathrm{~min}$ at $72{ }^{\circ} \mathrm{C}$. Amplified products were electrophoresed with $1.5 \%$ agarose gel and visualized with a UV illuminator.

The amplified fragments with expected size were purified by a commercial gel extraction kit (DF300, Qiagen), which was performed with an automated sequencing(Mission Biotech Co., Taipei, Taiwan). The sequences obtained from a NCC were initially compared with a canine $c$-kit sequence [GenBank: AF448148]. Sequences of HSAs were aligned with those of NCC using the BLAST program on the NCBI.

\section{Cloning of PCR amplicon containing the exon 9 region of c-kit}

To clarify sequences of the most 3 'end of $c$-kit exon 9 of HSAs, DNA fragments containing exon 9 were amplified by PCR with primers set EX9F/R (Table 2) and were then purified. Ligation was conducted by a TOPO TA Cloning Kit (450641, Invitrogen) followed by transforming to TOP10 competent cells and plating onto an LB
Table 2 Sequences and locations of c-kit primers used in this study

\begin{tabular}{|c|c|c|}
\hline Designation & Sequence $\left(5^{\prime}-3^{\prime}\right)$ & Primer location \\
\hline $1 \mathrm{~F}$ & CGATGAGAGGCGCTCGC & $27-34$ \\
\hline $1 \mathrm{R}$ & GGCGTAACACA TGAACACTCCAG & $891-913$ \\
\hline $2 \mathrm{~F}$ & GCTGGCATCATGGTGACTTC & $819-838$ \\
\hline $2 \mathrm{R}$ & CATGGGTTTCTGTAGATACTTGTAGG & $1668-1693$ \\
\hline $3 F$ & CACACCTTTGCTGATTGGCT & $1591-1610$ \\
\hline $3 R$ & GATTCGACCATGAGTAAGGAGG & $2419-2440$ \\
\hline $4 \mathrm{~F}$ & GGGTATGGCATTCCTGGC & $2359-2376$ \\
\hline $4 \mathrm{R}$ & GCTTCACACATCTTCGTGTACCA & $2937-2959$ \\
\hline $\mathrm{AF}$ & GCTCAGAGTCTATCGCAGCCACCG & $3-26$ \\
\hline$A R$ & CTGCCTTCTCTGTGATCCATTCGTTG & $271-296$ \\
\hline BF & GCTGTCCAAGAAATTCACCCTG & $619-640$ \\
\hline BR & ATATTACTTTCATTGTCAGACTTGGG & $1124-1149$ \\
\hline CF & AAAACTCGTCTCTGTCACCGTCTG & $1422-1445$ \\
\hline$C R$ & GATCTCCTCAACAACCTTCCACTG & $1691-1714$ \\
\hline DF & AAATCAGAGTTAATAGTCAGTGTCGG & $133-158$ \\
\hline DR & TTTATCCACATCGAGTCCACG & $729-749$ \\
\hline EX9F & CAACAATGTAGGCAGGAGTTCTG & $1495-1517$ \\
\hline EX9R & CAGCAAAGGTGTGAACAGGG & $1572-1591$ \\
\hline
\end{tabular}

$F$ forward, $R$ reverse

Annealing temperature dependent on combination of the primers: $55{ }^{\circ} \mathrm{C}$ for $1 \mathrm{~F}$ and $1 \mathrm{R}, 56{ }^{\circ} \mathrm{C}$ for $2 \mathrm{~F}$ and $2 \mathrm{R}, 52{ }^{\circ} \mathrm{C}$ for $3 \mathrm{~F}$ and $3 \mathrm{R}, 61^{\circ} \mathrm{C}$ for $\mathrm{AF}$ and $\mathrm{AR}, 54{ }^{\circ} \mathrm{C}$ for $\mathrm{BF}$ and $\mathrm{BR}, 57^{\circ} \mathrm{C}$ for $\mathrm{CF}$ and $\mathrm{CR}, 54^{\circ} \mathrm{C}$ for $\mathrm{DF}$ and $\mathrm{DR}, 57{ }^{\circ} \mathrm{C}$ for $\mathrm{AF}$ and $\mathrm{BR}$, $55{ }^{\circ} \mathrm{C}$ for P2F and $\mathrm{CR}, 56{ }^{\circ} \mathrm{C}$ for $\mathrm{CF}$ and P3R, and $54{ }^{\circ} \mathrm{C}$ for EX9F and EX9R

plate containing ampicillin as well as X-gal and IPTG for the case of blue/white screening.

After $16 \mathrm{~h}$ of incubation, 10 to 14 white colonies randomly picked from each plate were cultured at $37{ }^{\circ} \mathrm{C}$ overnight and then the plasmid DNA was extracted via High-Speed Plasmid Mini Kit, (PD300, Geneaid). Plasmids with insertion of $c$-kit DNA were initially confirmed by the digestion pattern using are striction enzyme EcoRI (New England Biolabs Inc.) followed by automated sequencing (Mission Biotech Co.).

\section{Statistical analysis}

The statistical analysis was performed using Statistical Package for the Social Science (Version 19.0, SPSS Taiwan Corp). Univariate logistic regression was used to establish the correlation between variables and KIT immunolabeling patterns. $P$-value $<0.05$ indicated a statistically significant difference between categorized groups.

\section{Results}

Animals

As listed in Additional file 1: Table S1, of the 16 dogs with HAs, seven were male and nine were female. Dogs with HSAs included 24 males and 12 females. Pedigree dogs comprised eight breeds and accounted for $56.3 \%$ $(9 / 16)$ of dogs with HAs, and $58.3 \%(21 / 36)$ of dogs 
with HSAs, which included Maltese $(n=3)$, Pomeranian $(n=2)$, and Golden Retriever $(n=2)$ with HAs, and Golden Retriever $(n=8)$, Miniature Schnauzer $(n=5)$, Beagle $(n=2)$, Maltese $(n=2)$, and Labrador Retriever $(n=2)$ with HSAs. Labrador retriever and Shetland sheepdog each accounted for 1/16 with HA; and Caucasian sheepdog and Welsh Corgi each accounted for 1/36 with HSA. The other $43.7 \%(7 / 16)$ of HA and $41.7 \%$ $(15 / 36)$ of HSA occurred in mixed dogs. The median age in dogs with HA was 11.8 years (range $2-16$ years), and 13.4 years (range 4-18 years) in dogs with HSA.

Clinical stages of HSA included stage III in $58.3 \%$ $(21 / 36)$, stage II in $22.2 \%(8 / 36)$, and stage I in $19.5 \%(7 / 36)$ of dogs. Histological grades were assigned as grade I in $5.6 \%(2 / 36)$, grade II in $33.3 \%$ $(12 / 36)$, and graded III in $61.1 \%(22 / 36)$ of dogs.

\section{Detection of KIT expression by IHC}

To study the correlation of KIT expression with $c$-kit sequence mutations in canine HSAs, initially, KIT expression in canine HSAs and KIT expression in HAs were detected by IHC analysis, of which, KIT staining was optimized using NCC as a positive control (Fig. 1a), and omitting KIT antibody as a negative control. As listed in Table 3, vWF expression was demonstrated in all HSAs (36/36, $100 \%$ ), vimentin expression was shown in $97.2 \%$ of HSAs (35/36), and KIT expression was detected in $94.4 \%$ of HSAs (34/36), whereas negative KIT expression was found in all HAs $(16 / 16,100 \%$, and Fig. 1b). All KIT-positive HSAs (34/34, $100 \%$ ) showed a diffuse cytoplasmic immunostaining pattern (Fig. 1 panels c-d).

\section{Identification of $c$-kit gene sequences of HSA}

In order to identify possible mutations of the $c$-kit gene in canine HSAs, amplicons covering the entire coding region of the $c$-kit gene were amplified by PCR using several sets of primers followed by automated sequencing. Initially, sequences representing wild type c-kit gene were amplified from a NCC. Compared with the $c$ kit coding sequences [GenBank: AF448148], a G to A transversion $\left(\mathrm{G}^{1275} \mathrm{~A}\right)$, a silent mutation at codon 425 of KIT, was identified in our NCC sample.

Next, the full length $c$-kit coding region of 12 HSAs with high KIT expression level (samples were indicated in Additional file 1: Table S1) was amplified for sequence identification. Compared with the wild-type c-kit sequences, the 1275 nucleotide (nt) was either A or G; $50 \%(6 / 12)$ of HSAs harbored the $\mathrm{A}^{1275} \mathrm{G}$ substitution. In addition, $8.3 \%(1 / 12)$ of HSA samples contained 3 other genetic variations at $\mathrm{C}^{159} \mathrm{~T}$ (codon 53 ), $\mathrm{C}^{414} \mathrm{~T}$ (codon 138), and $\mathrm{A}^{507} \mathrm{G}$ (codon 169) of c-kit. Overall, these four point mutations were silent mutations that did not alter amino acids.

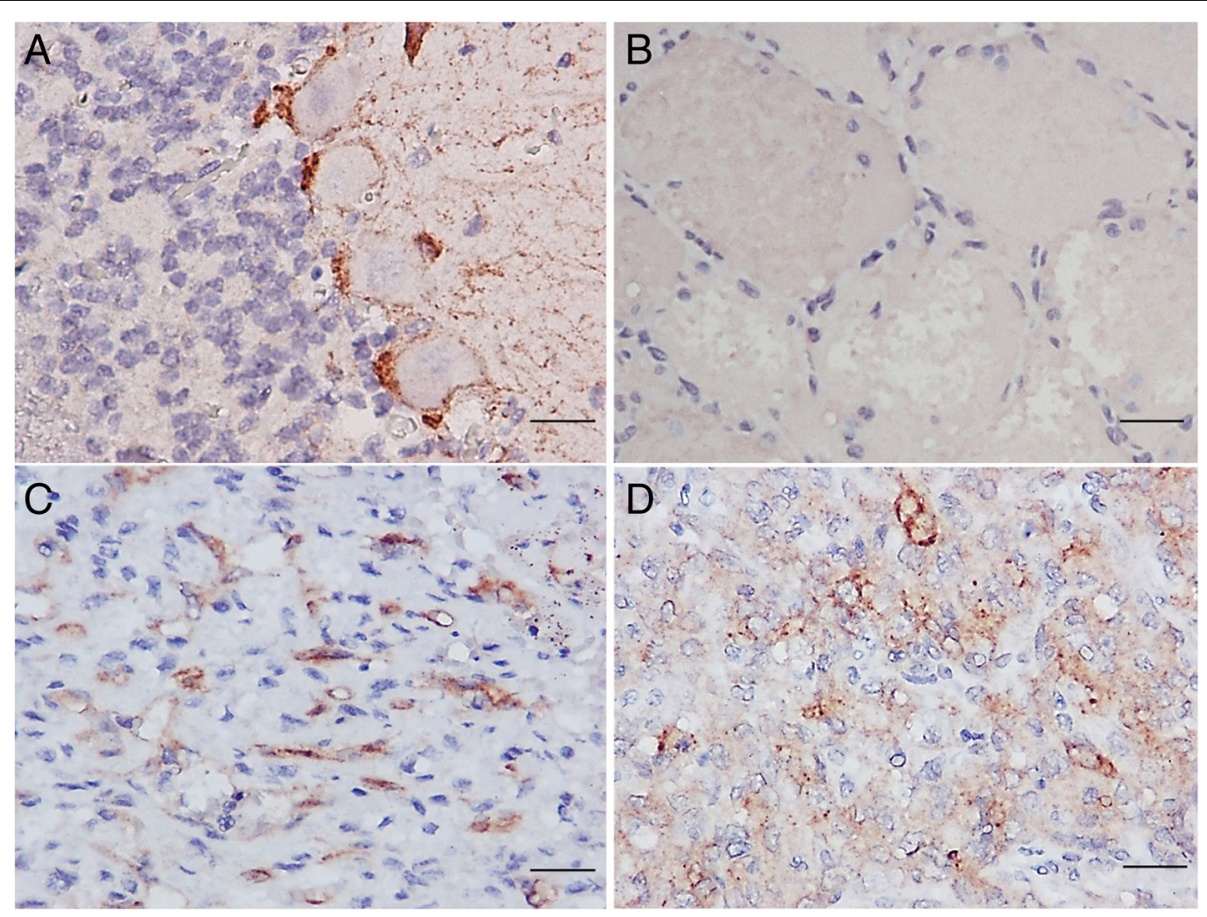

Fig. $1 \mathrm{Immunohistochemical} \mathrm{staining} \mathrm{for} \mathrm{KIT.} \mathrm{a} \mathrm{Strong} \mathrm{immunolabelling} \mathrm{in} \mathrm{the} \mathrm{cytoplasm} \mathrm{of} \mathrm{Purkinje} \mathrm{cells} \mathrm{of} \mathrm{a} \mathrm{NCC} \mathrm{was} \mathrm{used} \mathrm{as} \mathrm{a}$ positive control for KIT immunostaining. b Expression of KIT was not detected in a HA. c Strong immunoreactivities were observed inasplenic HSA with stage III and grade 3. d Positive immunoreactivities were observed ina cutaneous HSA with stage III and grade 2. Sections counterstained with hematoxylin. Bar $=50 \mu \mathrm{m}$ 
Table $3 \mathrm{IHC}$ analysis of VWF, vimentin and KIT expression in canine hemangiomas and hemangiosarcomas

\begin{tabular}{|c|c|c|c|c|c|c|c|c|c|c|c|}
\hline & \multirow[b]{2}{*}{ Number } & \multicolumn{3}{|c|}{ Intensity (\%) } & \multicolumn{3}{|c|}{ Positively tumor cells (\%) } & \multicolumn{2}{|c|}{ Immunoreactivity } & \multirow{2}{*}{$\begin{array}{c}\text { Chi- } \\
\text { square }\end{array}$} & \multirow[t]{2}{*}{$P$ value } \\
\hline & & Weak & Moderate & Strong & $<10 \%$ & $10-50 \%$ & $>50 \%$ & Positive & Negative & & \\
\hline $\begin{array}{l}\text { UWF for } \\
\text { HSAs }\end{array}$ & 36 & $0(0 \%)$ & $8(22.2 \%)$ & $28(77.8 \%)$ & $5(13.9 \%)$ & $7(19.4 \%)$ & $24(66.7 \%)$ & $36(100 \%)$ & $0(0 \%)$ & - & - \\
\hline $\begin{array}{l}\text { Vimentin } \\
\text { for HSAs }\end{array}$ & 36 & $1(2.8 \%)$ & $2(5.6 \%)$ & $33(91.6 \%)$ & $4(11.1 \%)$ & $9(25 \%)$ & $23(63.9 \%)$ & $35(97.2 \%)$ & $1(2.8 \%)$ & - & - \\
\hline \multirow[t]{2}{*}{$\begin{array}{l}\text { KIT for } \\
\text { HAs }\end{array}$} & 16 & $16(100 \%)$ & $0(0 \%)$ & $0(0 \%)$ & $16(100 \%)$ & $0(0 \%)$ & $0(0 \%)$ & $0(0 \%)$ & $16(100 \%)$ & & \\
\hline & & & & & & & & & & 43.654 & $<0.0001$ \\
\hline $\begin{array}{l}\text { KIT for } \\
\text { HSAs }\end{array}$ & 36 & $2(5.6 \%)$ & $9(25 \%)$ & $25(69.4 \%)$ & $6(16.7 \%)$ & $10(27.8 \%)$ & $20(55.5 \%)$ & $34(94.4 \%)$ & $2(5.6 \%)$ & & \\
\hline
\end{tabular}

VWF von Willebrand factor, HSAs hemangiosarcomas, HAs hemangiomas, Weak staining much weaker than positive control or negative, Moderate staining slightly weaker than positive control, Strong staining equal to positive control. -, not done. $P$ values $<0.05$ indicate significant difference

Interestingly, as judged by sequencing chromatogram, overlapping noise signals starting from codon 513 to 516 (the most 3' end) of the $c$-kit exon 9 were found in all DNA fragments amplified from HSAs (12/12, $100 \%)$ (Fig. 2a, left panel), indicating the presence of sequence polymorphisms. This phenomenon was not found in those amplified from NCCs (Fig. 2a, right panel). Isolation of the PCR amplicons containing this region revealed two distinct products (Fig. 2b, indicated as arrowheads). Sequence alignment indicated that 12 nucleotides, coding for residues 513-516 (GNSK), were deleted from the exon 9 variant, as compared with that of wild type (Fig. 2c).

\section{Investigation of sequence variations in exon 9 of c-kit}

Based on the migration pattern of gel electrophoresis, two types of exon 9 amplicons were present in all HSA samples (12/12), and in $83.3 \%(5 / 6)$ of NCCs (Fig. 3a). It is worthy of noting that the 12 nt deletion variant, designated GNSK-deletion herein, was the major product in HSAs samples that is distinct from normal canine tissues, which is consistent with sequencing chromatogram as indicated in Fig. 2a. Next, we investigated the correlation of the c-kit GNSK-deletion variant with canine HSAs.

To this end, we determined the approximate frequency of these two genotypes of $c$-kit exon 9 by cloning strategies [28]. DNA amplicons were cloned into TA vector following PCR, and more than ten bacterial colonies with $c$-kit exon 9 insertion randomly picked from individual cases were sequenced. In total, sequences of 140 clones containing exon 9, amplified from 12 HSA patients and 64 clones from 6 healthy canine cerebellums, were determined. The frequency of two $c$-kit exon

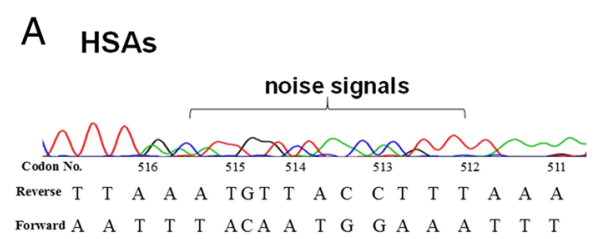

NCC

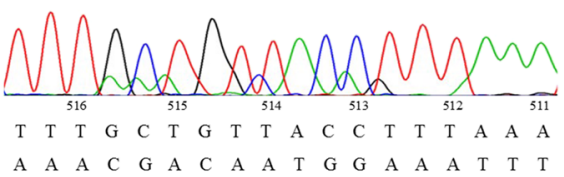

B

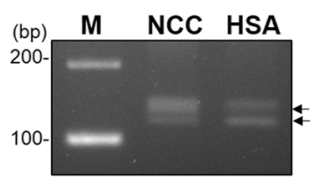

C

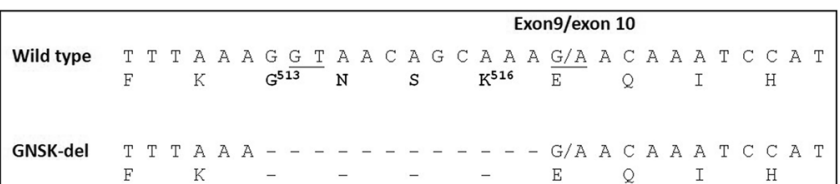

Fig. 2 Identification of sequence variations inc-kittranscript of canine HSAs. Following PCR amplification, sequences of amplicons were revealed by automated sequencing. a Distinct sequencing chromatograms of $c-k i t$ exon 9 were revealed between NCCand HSA samples. $\mathbf{b}$ The PCR products containing c-kit exon 9 were amplified from a NCCor a HSA and resolved in 3 \% agarose gel. M, 100-bp DNA size markers. c Sequence alignment of two c-kit exon 9 variants. Dashed lines indicate deletion of nucleotides. Underlines indicate the deduced conserved di-nucleotide of splicing donors and acceptor sites 

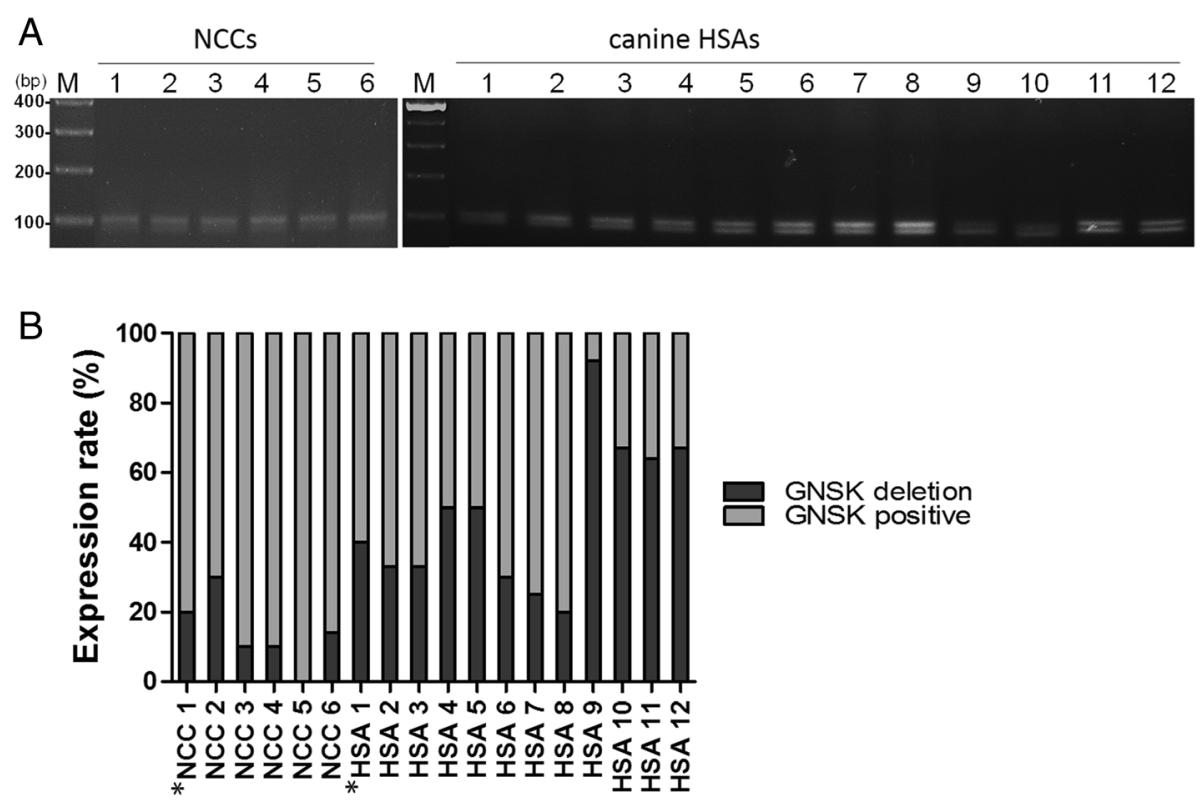

Fig. 3 The frequency of c-kit exon 9 variants in canine tissues. a PCR products containing the exon 9 region amplified from 6 healthy canine cerebellums (left panel) or 12 canine HSAs (right panel) were electrophoresed with $3 \%$ agarose gel. b The approximate frequency of the c-kit exon 9 variants was determined by cloning strategy. Numbers of colonies containing genotype representing wild type (grey bar), or 12-nt del (black bar) of exon 9 were summarized

9 genotypes in each sample are shown in Fig. 3b. Overall, 72 colonies from HSAs and 55 colonies from NCCs resembled wild-type $c$-kit exon 9 sequences, whereas 68 clones from HSAs and 9 clones from NCCs were the GNSK-deletion. Overall, frequency of this c-kit RNA variant in HSAs was $48.6 \%$ (68/140), significantly higher than $14.1 \%(9 / 64)$ of NCCs $(P=0.004)$.

\section{Discussion}

The expression profile and the mutation status of KIT have been intensively studied in human tumors, in particular, GISTs and MCTs [29]. Mutational hot spots in different regions of the $c$-kit gene have been found in human tumors. However, little is known about the $c$-kit mutation status of canine tumors. In the present study, the frequency and location of c-kit gene mutations of canine HSA was investigated. We analyzed the entire coding region of the $c$-kit transcript and revealed a variant with a 12-nt deletion in exon 9. Previously, the GNSK deletion was detected in $\mathrm{C} 2$ canine malignant mast cell line, but not in NCC [30]. In addition, while preparing this manuscript, $c$-kit sequences of canine HSA was revealed [17]. In that study, Gramer et al., referred GNSK insertion (the full length $c$-kit) was not amplified in canine HSA, indicating GNSK-deletion allele possibly is the major transcript that is consistent with our findings. We further demonstrated, in fact, both wild type (full length) and GNSK-deletion genotypes were present in canine tumor samples.
Interestingly, GNSK- deletion was present in a significantly higher level in canine HSAs as compared with NCCs.

It is worthy of noting that previously identified high frequency mutations were not present in canine HSAs; for instance, mutations in exon 11 [31], and alterations in exon 9 of $c$-kit including SNPs and duplication in human GISTs [32, 33], and SNPs (missense mutations; $\mathrm{S}^{479} \mathrm{I}, \mathrm{N}^{508}$ I substitution) in canine MCTs [7]. Interestingly, the GNSK-deletion presents not only in HSAs, but also in NCCs (to a much lower frequency); this variation was regarded as a c-kit isoform. Deletion of 12-nt (GGTAACAGCAAA, coding for $\mathrm{G}^{513} \mathrm{NSK}^{516}$ residues) has not been reported in previous studies on canine GISTs and MCTs [31, 34]. Based on the signal intensity of sequence chromatography and also the frequency estimated from TA cloning strategy, we suspect the two genotypes (wild type and GNSK-deletion) of KIT were expressed at similar levels in canine HSAs.

Furthermore, as GNSK-del mutation of exon 9 was not detected in genomic DNA of all samples, the presence of two $c$-kit RNAs is very likely due to alternative splicing during processes of pre-mRNA. Indeed, the deleted sequences (5'-GGTAACAGCAAAG-3') in exon 9 comprised consensus splicing signals [35], i.e. the two nucleotides in italic font, of which the GT di-nucleotide at the $5^{\prime}$ end, and AG at the3' end (G residue is the first nucleotide in the adjacent exon 10) serves as a donor site and acceptor site of the splicing event, respectively. 
Therefore, the presence of GT and AG di-nucleotides in the end of exon 9 would act as an alternative splicing signal leading to deletion of the 12 nucleotides.

In previous studies, the isoform with deletion of a tetrapeptide sequence (GNNK) in the extracellular juxtamembrane region of KIT has been demonstrated in mice and humans [36, 37]. While these two KIT isoforms share similar affinity to ligand SCF [24], expression of KIT with GNNK deletion induced stronger transformation effects in NIH3T3 cells and was more tumorigenic in nude mice, as compared with wild type KIT [38]. The underlying mechanism has been proposed; the GNNK negative isoform can be phosphorylated (on tyrosine) at a higher level and more rapidly than the GNNK-positive isoform upon SCF ligand binding that leads to stimulating a stronger downstream signaling [39]. Additionally, the GNNK negative isoform was more resistant to tyrosine kinase inhibitor when compared with the GNNK positive isoform in the absence of human SCF [40]. Moreover, it is worthy of noting that genotyping in this study revealed the presence of GNSK-deletion in NCCs, although to a lesser extent than in canine HSAs. This is in line with the results of IHC, in which malignant HSAs and NCCs expressed high levels of KIT. Expression of KIT in the cerebellum implicates the importance of KIT in maintenance of cerebellar functionality. Indeed, embryonic knockdown expression of KIT in the rat cortex indicates KIT participates in radial migration of cortical neurons and also in the correct formation of callosal projection neurons [41]. In addition, as seen in Fig. 3, the ratio of GNSK-deletion of $c$-kit transcripts in HSAs was significantly higher than that in NCCs, indicating the role that the GNSK-deletion isoform plays in the development of HSAs, possibly due to activating downstream signaling of KIT.

The results of IHC demonstrated that KIT is predominantly expressed in malignant canine HSAs while it is absent in benign HAs. This finding is consistent with one previous study [8]. In addition, expression of KIT in all canine HSAs displayed diffuse cytoplasmic staining, even in HSAs with various histologic grades. According to the definition of a previous report, the immunostaining pattern of KIT in canine HSAs was classified into pattern III [42]. It has been reported that pattern III is significantly associated with a high histologic grade, poor differentiation [43], local recurrence, and metastasis [42].

Although most HSAs expressed high levels of KIT, the downstream signal transduction pathways activated by KIT in canine HSAs remain unclear. The active and dysregulated dimeric KIT may result in neoplastic transformation of normal cells overexpressing KIT [13]. The constitutional phosphorylation of KIT and activations of the downstream signaling pathways including the MAPK pathway, PI3K/AKT/mTOR pathway and JAK/STAT pathway have been reported $[41,44,45]$. Therefore, the role of GNSK-deletion KIT in the activation of downstream signaling pathways in canine HSAs or in the pathogenesis of canine HSAs warrants further investigation.

\section{Conclusions}

The positive immunoactivity of KIT was demonstrated in the majority of HSAs, while it was not detectable in HAs. Hence, expression of KIT might be used as a marker to distinguish benign or malignant vascular endothelial originated tumors. Sequencing of the entire coding region indicated previously reported sequence variations were not present in canine HSAs analyzed in this study. Instead, a KIT isoform with deletion of GNSK, generated by alternative splicing, was detected in all canine HSAs tested. Moreover, a significantly higher proportion of this GNSK-deletion isoform in canine HSAs than in normal cerebellum was demonstrated for the first time.

\section{Additional file}

Additional file 1: Table S1. Summary of clinical characteristics of dogs with HSA or HA, and IRS of KIT expression profile. (DOCX $17 \mathrm{~kb}$ )

\section{Abbreviations}

GIST, gastrointestinal stromal tumors; HA, hemangioma; HSA, hemangiosarcoma; IHC, immunohistochemistry; IRS, immunoreactive score; MCT, mast cell tumor; NCC, normal canine cerebellum; PP, percentage of positive cell; SCF, stem cell factor; SI, staining intensity; VWF, von Willebrand factor

\section{Acknowledgements}

All authors wish to thank Yi-Ping Yang for assisting in histopathological examination of all canine HAs and HSAs. Shin-Hung Huang for normal canine cerebellums collection.

\section{Funding}

No funding was obtained for this study.

Availability of data and materials

All the data supporting the findings of this study is contained within the manuscript. The Accession Number of reference gene was cited in this manuscript [GenBank: AF448148].

\section{Authors' contributions}

YCC conducted the experiments, analyzed the data, and prepared the manuscript. JWL evaluated the results of histopathological examinations and immunohistochemistry stains. WLH and SCC designed this research and revised the manuscript. All authors read and approved the final manuscript.

\section{Competing interests}

None of the authors of this paper has a financial or personal relationship with other people or organisations that could inappropriately influence or bias the paper content.

Consent for publication

Not applicable. 


\section{Ethics approval and consent to participate}

The samples of this study were obtained from dogs with HA or HAS by surgical excision or needle core biopsy for the main purpose of diagnosis and treatments. The remaining tissues were then also used for this research with agreement of every owners. The protocol of study was approved by the Institutional Animal Care and Use Committee of National Chung Hsing University [IACUC Number: 102-70].

\section{Author details}

'Department of Veterinary Medicine, College of Veterinary Medicine, National Chung Hsing University, 250 Kuo-Kuang Road, Taichung 40227, Taiwan. ${ }^{2}$ Graduate Institute of Veterinary Pathobiology, College of Veterinary Medicine, National Chung Hsing University, 250 Kuo-Kuang Road, Taichung 40227, Taiwan. ${ }^{3}$ Graduate Institute of Microbiology and Public Health, College of Veterinary Medicine, National Chung Hsing University, 250 Kuo-Kuang Road, Taichung 40227, Taiwan. ${ }^{4}$ Veterinary Medical Teaching Hospital, College of Veterinary Medicine, National Chung Hsing University, 250 Kuo-Kuang Road, Taichung 40227, Taiwan.

\section{Received: 8 April 2016 Accepted: 13 July 2016}

\section{Published online: 16 July 2016}

\section{References}

1. Brown NO, Patnaik AK, MacEwen EG. Canine hemangiosarcoma: retrospective analysis of 104 cases. J Am Vet Med Assoc. 1985;186(1):56-8.

2. Spangler WL, Culbertson MR. Prevalence, type, and importance of splenic diseases in dogs: 1,480 cases (1985-1989). J Am Vet Med Assoc. 1992;200(6): 829-34.

3. Dernell WS, Withrow SJ, Kuntz CA, et al. Principles of treatment for soft tissue sarcoma. Clin Tech Small Anim Pract. 1998;13(1):59-64.

4. Ogilvie GK, Powers BE, Mallinckrodt CK, et al. Surgery and doxorubicin in dogs with hemangiosarcoma. J Vet Intern Med. 1996;10(6):379-84.

5. Galli SJ, Zsebo KM, Geissler EN. The kit ligand, stem cell factor. Adv Immunol. 1994;55:1-96.

6. Vliagoftis H, Worobec AS, Metcalfe DD. The protooncogene c-kit and c-kit ligand in human disease. J Allergy Clin Immunol. 1997;100(4):435-40.

7. Miettinen M, Sarlomo-Rikala M, Lasota J. KIT expression in angiosarcomas and fetal endothelial cells: lack of mutations of exon 11 and exon 17 of C-kit. Mod Pathol. 2000;13(5):536-41.

8. Sabattini S, Bettini G. An immunohistochemical analysis of canine haemangioma and haemangiosarcoma. J Comp Pathol. 2009;140(2-3):158-68.

9. Kessler M, Maurus Y, Kostlin R. Hemangiosarcoma of the spleen: clinical aspects in 52 dogs. Tierarztl Prax Ausg K Kleintiere Heimtiere. 1997;25(6):651-6.

10. Hirota S, Isozaki K, Moriyama Y, et al. Gain-of-function mutations of c-kit in human gastrointestinal stromal tumors. Science. 1998;279(5350):577-80.

11. Moskaluk CR, Tian Q, Marshall CR, et al. Mutations of c-kit JM domain are found in a minority of human gastrointestinal stromal tumors. Oncogene. 1999:18(10):1897-902.

12. Nakahara M, Isozaki K, Hirota S, et al. A novel gain-of-function mutation of c-kit gene in gastrointestinal stromal tumors. Gastroenterology. 1998;115(5):1090-5.

13. Kitamura Y, Tsujimura T, Jippo T, et al. Regulation of development, survival and neoplastic growth of mast cells through the c-kit receptor. Int Arch Allergy Immunol. 1995;107(1-3):54-6.

14. Roskoski Jr R. Structure and regulation of Kit protein-tyrosine kinase-the stem cell factor receptor. Biochem Biophys Res Commun. 2005;338(3):1307-15.

15. Debiec-Rychter M, Dumez H, Judson I, et al. Use of C-KIT/PDGFRA mutational analysis to predict the clinical response to imatinib in patients with advanced gastrointestinal stromal tumours entered on phase I and II studies of the EORTC Soft Tissue and Bone Sarcoma Group. Eur J Cancer. 2004:40(5):689-95.

16. Kobayashi M, Kuroki S, Ito K, et al. Imatinib-associated tumour response in a dog with a non-resectable gastrointestinal stromal tumour harbouring a c-kit exon 11 deletion mutation. Vet J. 2013;198(1):271-4

17. Gramer I, Kessler M, Geyer J. Detection of novel polymorphisms in the ckit gene of canine patients with lymphoma, melanoma, haemangiosarcoma, and osteosarcoma. Vet Res Commun. 2016. [Epub ahead of print].

18. Frost MJ, Ferrao PT, Hughes TP, et al. Juxtamembrane mutant V560GKit is more sensitive to Imatinib (STI571) compared with wild-type c-kit whereas the kinase domain mutant D816VKit is resistant. Mol Cancer Ther. 2002;1(12):1115-24.

19. Miettinen M, Lasota J. KIT (CD117): a review on expression in normal and neoplastic tissues, and mutations and their clinicopathologic correlation Appl Immunohistochem Mol Morphol. 2005;13(3):205-20.
20. Wood CA, Moore AS, Gliatto JM, et al. Prognosis for dogs with stage or II splenic hemangiosarcoma treated by splenectomy alone: 32 cases (1991-1993). J Am Anim Hosp Assoc. 1998;34(5):417-21.

21. Kuntz CA, Dernell WS, Powers BE, et al. Prognostic factors for surgical treatment of soft-tissue sarcomas in dogs: 75 cases (1986-1996). J Am Vet Med Assoc. 1997;211(9):1147-51.

22. Hellstrom S, Tengblad A, Johansson C, et al. An improved technique for hyaluronan histochemistry using microwave irradiation. Histochem J. 1990; 22(12):677-82.

23. Meis JM, Enzinger FM. Chondroid lipoma. A unique tumor simulating liposarcoma and myxoid chondrosarcoma. Am J Surg Pathol. 1993;17(11):1103-12.

24. London CA, Kisseberth WC, Galli SJ, et al. Expression of stem cell factor receptor (c-kit) by the malignant mast cells from spontaneous canine mast cell tumours. J Comp Pathol. 1996;115(4):399-414.

25. Shyu RY, Jiang SY, Chou JM, et al. RARRES3 expression positively correlated to tumour differentiation in tissues of colorectal adenocarcinoma. $\mathrm{Br} \mathrm{J}$ Cancer. 2003;89(1):146-51.

26. Reguera MJ, Rabanal RM, Puigdemont A, et al. Canine mast cell tumors express stem cell factor receptor. Am J Dermatopathol. 2000;22(1):49-54.

27. Ma Y, Longley BJ, Wang $X$, et al. Clustering of activating mutations in C-KIT's juxtamembrane coding region in canine mast cell neoplasms. J Invest Dermatol. 1999:112(2):165-70.

28. Lacey EA. Microsatellite variation in solitary and social tuco-tucos: molecular properties and population dynamics. Heredity. 2001;86(Pt5):628-37.

29. Cruse G, Metcalfe DD, Olivera A. Functional deregulation of KIT: link to mast cell proliferative diseases and other neoplasms. Immunol Allergy Clin North Am. 2014;34(2):219-37.

30. London CA, Galli SJ, Yuuki T, et al. Spontaneous canine mast cell tumors express tandem duplications in the proto-oncogene c-kit. Exp Hematol. 1999;27(4):689-97.

31. Letard S, Yang Y, Hanssens $K$, et al. Gain-of-function mutations in the extracellular domain of KIT are common in canine mast cell tumors. Mol Cancer Res. 2008;6(7):1137-45.

32. Lux ML, Rubin BP, Biase TL, et al. KIT extracellular and kinase domain mutations in gastrointestinal stromal tumors. Am J Pathol. 2000;156(3):791-5.

33. Martin J, Poveda A, Llombart-Bosch A, et al. Deletions affecting codons 557-558 of the c-KIT gene indicate a poor prognosis in patients with completely resected gastrointestinal stromal tumors: a study by the Spanish Group for Sarcoma Research (GEIS). J Clin Oncol. 2005;23(25):6190-8.

34. Gregory-Bryson E, Bartlett E, Kiupel M, et al. Canine and human gastrointestinal stromal tumors display similar mutations in c-KIT exon 11. BMC Cancer. 2010;10:559.

35. Mount SM. A catalogue of splice junction sequences. Nucleic Acids Res. 1982;10(2):459-72.

36. Crosier PS, Ricciardi ST, Hall LR, et al. Expression of isoforms of the human receptor tyrosine kinase c-kit in leukemic cell lines and acute myeloid leukemia. Blood. 1993;82(4):1151-8.

37. Hayashi $\mathrm{S}$, Kunisada $\mathrm{T}$, Ogawa M, et al. Exon skipping by mutation of an authentic splice site of c-kit gene in W/W mouse. Nucleic Acids Res. 1991; 19(6):1267-71.

38. Caruana G, Cambareri AC, Ashman LK. Isoforms of c-KIT differ in activation of signalling pathways and transformation of NIH3T3 fibroblasts. Oncogene. 1999;18(40):5573-81.

39. Voytyuk O, Lennartsson J, Mogi A, et al. Src family kinases are involved in the differential signaling from two splice forms of c-Kit. J Biol Chem. 2003; 278(11):9159-66

40. Chan EC, Bai Y, Bandara G, et al. KIT GNNK splice variants: expression in systemic mastocytosis and influence on the activating potential of the D816V mutation in mast cells. Exp Hematol. 2013:41(10):870-81.

41. Guijarro $P$, Wang $Y$, Ying $Y$, et al. In vivo knockdown of cKit impairs neuronal migration and axonal extension in the cerebral cortex. Dev Neurobiol. 2013; 73(12):871-87.

42. Thompson JJ, Yager JA, Best SJ, et al. Canine subcutaneous mast cell tumors: cellular proliferation and KIT expression as prognostic indices. Vet Pathol. 2011;48(1):169-81.

43. Gil da Costa RM, Matos E, Rema A, et al. CD117 immunoexpression in canine mast cell tumours: correlations with pathological variables and proliferation markers. BMC Vet Res. 2007:3:19.

44. Ronnstrand L. Signal transduction via the stem cell factor receptor/c-Kit. Cell Mol Life Sci. 2004;61(19-20):2535-48.

45. Adachi M, Hoshino Y, Izumi Y, et al. Immunohistochemical detection of a potential molecular therapeutic target for canine hemangiosarcoma. J Vet Med Sci. 2016;78(4):649-56. 